\title{
La carrière de Mei Wending (1633-1721) et le statut des sciences mathématiques dans le savoir lettré
}

Mei Wending's (1633-1721) Career and the Status of the Mathematical Sciences in Scholarly Learning 梅文鼎 (1633-1721) 的學術生涯與作為士人之學的曆算的建構

\section{Catherine Jami}

\section{(2) OpenEdition}

Journals

Édition électronique

URL : http://journals.openedition.org/extremeorient/305

DOI : 10.4000/extremeorient.305

ISSN : 2108-7105

Éditeur

Presses universitaires de Vincennes

\section{Édition imprimée}

Date de publication : 31 décembre 2013

Pagination : 19-47

ISBN : 978-2-84292-404-1

ISSN : 0754-5010

Référence électronique

Catherine Jami, «La carrière de Mei Wending (1633-1721) et le statut des sciences mathématiques dans le savoir lettré », Extrême-Orient Extrême-Occident [En ligne], 36 | 2013, mis en ligne le 01 juin 2017, consulté le 21 avril 2019. URL : http://journals.openedition.org/extremeorient/305 ; DOI : 10.4000/extremeorient.305 


\title{
La carrière de Mei Wending (1633-1721) et le statut des sciences mathématiques dans le savoir lettré
}

\author{
Catherine Jami
}

Alors que les mathématiques et l'astronomie sont toujours restées marginales dans les examens de la fonction publique impériale chinoise, les $\mathrm{XVII}^{\mathrm{e}}$ et $\mathrm{XVIII}^{\mathrm{e}}$ siècles ont vu l'intégration progressive de ces deux disciplines aux savoirs lettrés ${ }^{1}$. Nul n'incarne mieux ce processus que Mei Wending (1633-1721), l'auteur le plus prolifique de cette époque dans ces domaines. Son œuvre comporte près d'une centaine de titres, de longueur variable, presque tous consacrés aux mathématiques ou à l'astronomie, et dont seule une partie est aujourd'hui conservée ${ }^{2}$. L'objectif de cet article est de retracer la carrière de Mei Wending pour montrer que sa mobilité géographique sur le territoire impérial dessine un itinéraire caractéristique de lettré: il s'agit aussi de montrer comment, en retour, les principaux lieux où il a travaillé ont donné leur dynamique à sa carrière et à son œuvre.

Mei Wending a vécu dans sa jeunesse la transition dynastique des Ming (1368-1644) aux Qing (1644-1911): sa vie a donc pour cadre historique la conquête de la Chine par les Mandchous et la consolidation de la dynastie par l'empereur Kangxi (r. 1662-1722); les deux derniers tiers de la vie de Mei, soit près de soixante ans, se sont déroulés sous le règne de cet empereur. Celuici s'est notamment efforcé de se concilier les élites lettrées parmi lesquelles étaient recrutés les fonctionnaires qui administraient l'empire sous les Ming, dont les Qing reprennent les principales institutions. Ainsi, après les troubles et parfois les ravages de la guerre de conquête, les examens qui servent à ce recrutement sont remis en place très rapidement. Dans le discours officiel, les Qing ont reçu le mandat céleste et sont donc les successeurs légitimes

1. Elman 2000 : 481-485; Jami 2012 : 280-283.

2. Outre les deux collections de ses œuvres rassemblées au XVIII ${ }^{\mathrm{e}}$ siècle (Wu'an lisuan quanshu, 1721, et Meishi Congshu jiyao, 1761), on conserve aujourd'hui de rares manuscrits et éditions de certains de ses ouvrages imprimées de son vivant. 
des Ming. Cependant, le sentiment loyaliste envers la dynastie déchue, particulièrement vif au Jiangnan, région dont est originaire Mei Wending, pousse certains savants à refuser d'entrer au service des Qing. C'est à la génération suivante que les Qing parviendront à se concilier les élites lettrées de cette région, qui est depuis le $\mathrm{x}^{\mathrm{e}}$ siècle le centre économique et intellectuel de la Chine. Dans ce but, Kangxi, premier souverain mandchou né à Pékin, met tout en œuvre pour incarner le monarque confucéen ; ainsi, tout en étudiant lui-même les classiques, il promeut l'école de Cheng Yi (1033-1107) et Zhu Xi (1130-1200), dont la philosophie est érigée depuis les Ming en orthodoxie impériale; l'empereur cultive aussi le patronage des activités savantes issues de la tradition chinoise.

Dans le domaine des sciences mathématiques, l'époque est caractérisée par l'introduction et l'appropriation de nouveaux savoirs. Les missionnaires jésuites qui sont entrés en Chine à la fin $\mathrm{du} \mathrm{XVI}^{\mathrm{e}}$ siècle ont introduit des éléments de ces sciences telles qu'elles étaient alors enseignées dans leurs collèges en Europe. En 1607, Matteo Ricci (1552-1610), le fondateur de la mission jésuite de Chine, et Xu Guangqi (1562-1633) publient une traduction des six premiers livres des Éléments de géométrie d'Euclide ${ }^{3}$; quelques années plus tard, en 1614, c'est un traité de calcul écrit, le Guide du calcul dans l'écriture commune (Tongwen suanzhi) qui est publié. Ces ouvrages ont un impact d'autant plus important qu'à l'époque certains des classiques mathématiques de la Chine ancienne et les grands ouvrages des $\mathrm{XIII}^{\mathrm{e}}$ et $\mathrm{XIV}^{\mathrm{e}}$ siècles (époque généralement considérée comme l'âge d'or des mathématiques en Chine) sont perdus. Plusieurs traités d'astronomie sont également publiés. En 1629, Xu Guangqi est chargé de réformer le calendrier impérial; sous sa direction, plusieurs missionnaires jésuites y travaillent, écrivant notamment une série de traités qui sont rassemblés sous le titre Livres calendaires de l'ère Chongzhen (Chongzhen lishu). Dès la prise de Pékin par les Mandchous en 1644, Johann Adam Schall von Bell (1592-1666), l'un des jésuites qui ont travaillé à la réforme du calendrier, fait allégeance aux nouveaux maîtres de la capitale et reçoit la charge de directeur du Bureau de l'astronomie (Qintianjian), entrant ainsi dans la fonction publique chinoise. Les méthodes introduites par les jésuites dans leurs écrits y sont désormais utilisées pour les calculs calendaires. Les Livres calendaires de l'ère Chongzhen, désormais appelés les Livres calendaires suivant la nouvelle méthode occidentale (Xiyang xinfa lishu), représentent l'astronomie impériale. Ces changements suscitent des controverses parfois violentes : en 1664, Schall est démis de ses fonctions à la suite d'accusations portées contre lui; tous les missionnaires sont alors expulsés des provinces.

3. Engelfriet 1998. 
Le rétablissement d'un jésuite, Ferdinand Verbiest (1623-1688), au Bureau de l'astronomie par le jeune Kangxi en 1669 permet la poursuite de l'activité missionnaire dans l'empire et remet les savoirs occidentaux à l'honneur. L'empereur lui-même se met alors à l'étude des sciences mathématiques, prenant Verbiest comme professeur ${ }^{4}$. Il demeure cependant une certaine hostilité envers les savoirs mis en œuvre par les jésuites parmi les lettrés et fonctionnaires, et une certaine méfiance envers les jésuites eux-mêmes parmi les savants qui, comme Mei Wending, reconnaissent la valeur des méthodes occidentales en sciences mathématiques. L'attitude des lettrés chinois vis-àvis des «savoirs occidentaux» (xixue) se comprend aussi à la lumière de leurs sentiments ambivalents à l'égard des conquérants mandchous.

Cet article s'appuie essentiellement sur les œuvres de Mei qui nous sont parvenues, sur une bibliographie de ses propres œuvres qu'il aurait compilée vers 1702 et qui a été revue et augmentée après sa mort ${ }^{5}$, sur l'anthologie de ses poèmes et de sa prose publiée par son petit-fils ${ }^{6}$, et sur les témoignages de lettrés qui l'ont connu. Ces sources sont par nature parcellaires, et éclairent davantage certains moments de la vie de Mei que d'autres; ainsi on sait peu de chose sur les quarante premières années. Une difficulté à laquelle on se heurte est la datation de ses ouvrages et de leur impression. En effet, très peu d'éditions anthumes semblent avoir été conservées; celles qui l'ont été n'ont pas forcément une préface écrite au moment de l'impression. Il faut aussi rappeler que le fait qu'un texte soit gravé sur des planches (rappelons que tous ces ouvrages sont xylographiés) ne nous dit rien sur le nombre d'exemplaires qui en ont été imprimés - ni sur la date à laquelle un exemplaire particulier a pu être fabriqué; la question se pose particulièrement pour les ouvrages savants tels que ceux de Mei Wending, qui relevaient de l'édition non commerciale. Notons au passage que ces sources - comme on peut s'y attendre - sont muettes sur la dimension économique de l'itinéraire de Mei: le coût d'un voyage, celui de la résidence en un lieu donné, d'un livre chez un libraire, voire du papier sur lequel on rédige ou recopie un ouvrage, ne seront donc pas abordés dans ce qui suit. Je m'appuie aussi sur un certain nombre de travaux concernant Mei

\footnotetext{
4. Jami 2012: 35-81.

5. Bibliographie des euvres astronomiques et mathématiques de Wu'an (Wu'an lisuan shumu). L'édition du Siku quanshu utilisée ici est intitulée Wu'an lisuan shuji; SKQS 795: 961-992. Elle comporte soixante-deux titres d'astronomie et vingt-six titres de mathématiques.

6. Mei 1995.
} 
Wending ${ }^{7}$, et plus particulièrement sur deux chronologies de sa vie (nianpu), en vérifiant autant que possible les citations qui y sont données ${ }^{8}$.

\section{Le district de Xuancheng}

De la jeunesse de Mei Wending, nous ne sommes informés que par quelques souvenirs évoqués par lui-même, par quelques phrases quelque peu emphatiques dans sa biographie rédigée en 1699 par Mao Qike et dans le Registre généalogique du clan Mei de Wenfeng (Wenfeng Meishi zongpu ${ }^{9}$. La famille Mei y aurait été présente depuis l'époque Song. La maison familiale se trouvait à la campagne; elle a été démolie au début des années 2000. Les tombes de Mei Wending et de ses frères, en revanche, ont été restaurées et se trouvent encore non loin de là, dans des champs qui appartenaient à la famille ${ }^{10}$.

Mei Wending est né en 1633 près de Xuancheng, siège de la préfecture de Ningguo, alors rattachée à la zone métropolitaine Sud (Nan Zhili) de la dynastie Ming; il était l'aîné d'une fratrie de quatre garçons nés de la même mère; tous ont reçu l'enseignement de leur père et de leur grand-père, versés dans les commentaires du Yijing de l'époque Song, dans la tradition des «figures et nombres» (xiangshu). Celle-ci s'intéressait aux régularités du cosmos, régularités dans lesquelles les nombres étaient supposés tenir une place importante; l'étude des relations entre les hexagrammes s'y rattache ${ }^{11}$. L'observation du ciel nocturne, dans lequel certaines de ces régularités sont visibles, faisait partie de cette culture familiale. Wending est reçu à l'examen de district en 1647 , un an après que la conquête mandchoue a ravagé la région ${ }^{12}$. Le rang de «talent cultivé» (xiucai) qu'il acquiert ainsi définit son identité : il est avant tout un lettré $(r u)$ dans la tradition confucéenne, avec les implications

7. Citons dans l'ordre chronologique de première publication: Li Yan 1998; Hashimoto 1970 et 1973; Martzloff 1981; Jami 1994, 2004 et 2012: chap. 4, 10 et 11; Han Qi 1996; Li Di 2006.

8. Li Yan 1998; Li Di 2006 : 19-55, 422-440.

9. Selon ce registre, le clan Mei serait originaire de Wenfeng au Henan. Li Di 2006 : 1920 .

10. Li Di, qui a visité cette maison en 1986, en a donné une description (Li Di 2006 : 22-24). En 2011, je me suis rendue à Xuancheng grâce à l'aide du Professeur Liu Dun (IHNS, CAS, Pékin); nous avons été informés sur place que la maison avait été démolie une dizaine d'années plus tôt, alors qu'un musée à la mémoire de Mei Wending (Mei Wending jinian guan) a été ouvert en ville.

11. Arrault 2002.

12. Wakeman $1985: 736$, n. 55. 
morales que cela comporte; c'est en tant que tel qu'il va se consacrer à des disciplines considérées par ses pairs comme purement techniques.

Vers 1660, il commence à étudier l'astronomie auprès de Ni Guanhu (1616- après 1695), un savant de Xuancheng dont on sait peu de chose, avec deux de ses frères: Wennai (1637-1671) et Wenmi (1641-1716). Le premier ouvrage de Wending, intitulé Propos superflus sur l'astronomie (Lixue pianzhi), résulte de la lecture qu'il fait avec ces derniers d'écrits astronomiques de leur maître; il est achevé en 1662. Dans les années qui suivent, Mei rédige un certain nombre de commentaires au fil de ses lectures, dont certaines sont des traités anciens.

Pendant la même période, il travaille également à deux ouvrages mathématiques: leurs titres citent les intitulés du premier et du huitième des «neuf chapitres» en lesquels étaient structurées les mathématiques dans la tradition chinoise ${ }^{13}$ : la Méthode générale des champs rectangulaires (Fangtian tongfa), préfacée par l'auteur en $1664^{14}$, et la Discussion sur les tableaux rectangulaires (Fangcheng lun). Le premier, très court, consiste en une liste de relations entre les nombres. Ces relations sont de deux types: celles définies par la cosmologie chinoise telle que la transmet la tradition des «figures et nombres», et les conversions des unités de mesure. Le second ouvrage, beaucoup plus volumineux (six chapitres), est consacré à ce qu'on appelle aujourd'hui les systèmes d'équations linéaires, qui, pour Mei Wending, est l'aboutissement et la synthèse de toutes les méthodes de calcul ${ }^{15}$. Sa lecture révèle notamment certains des ouvrages auxquels Mei a accès : la traduction des Éléments de 1607, et le Guide du calcul dans l'écriture commune, ainsi que divers traités de l'époque Ming, dont le fameux Lignage unifié des méthodes mathématiques (Suanfa tongzong, 1592), qui restera un best-seller jusqu'à la fin de la dynastie Qing. Ce dernier ouvrage consacre un chapitre aux «tableaux rectangulaires», tandis que le Guide du calcul donne une méthode censée permettre de résoudre tous les problèmes tels que le système d'équations linéaires. Mei est critique envers l'un et l'autre, à des degrés divers. Sous couvert de restaurer l'un des «neuf chapitres», Mei développe le traitement des systèmes d'équations linéaires avec une rigueur sans précédent, élaborant une classification des problèmes suivant le type d'opération à effectuer pour les résoudre ${ }^{16}$. Achevée en 1672, la Discussion est préfacée la même année par Pan Lei (1646-1708), un lettré du Jiangnan s'intéressant aux mathématiques,

13. Chemla et Guo 2004.

14. Li Yan 1998: 520; MSCSJY 5: 16a.

15. Jami 2012: 90-101.

16. Martzloff 1981 : 161-234; Jami 2012: 90-101. 
qui est dès cette époque en correspondance avec Mei; il est surtout connu comme disciple du savant Gu Yanwu (1613-1682) ${ }^{17}$.

L'année suivante, Mei Wending reçoit pour la première fois une commande ; cela nous indique qu'il était alors connu comme un spécialiste des phénomènes célestes à l'échelle locale. Shi Runzhang (1619-1683), haut fonctionnaire qui s'est alors retiré dans sa ville natale de Xuancheng, dirige la compilation de la Monographie de la Préfecture de Ningguo (Ningguo fuzhi); il demande à Mei d'en rédiger un chapitre, consacré à la «répartition des domaines » (fenye). Il s'agit d'une pratique particulière de l'astrologie, qui consiste à établir des corrélations entre les régions du ciel et les subdivisions d'un territoire; sur la base de ces corrélations, les phénomènes célestes observés sont alors interprétés comme des présages concernant la partie du territoire à laquelle correspond la région du ciel où ils sont observés. Cette pratique proprement territoriale de l'observation du ciel remonterait à l'Antiquité. Un autre traité du même type sera demandé à Mei quelques années plus tard pour la Monographie du district de Xuancheng (Xuancheng xianzhi, 1678); il reprend alors dans les grandes lignes ce qu'il a écrit pour la préfecture de Ningguo ${ }^{18}$.

Les travaux d'astronomie de Mei Wending écrits dans les quarante premières années de sa vie ont un point commun: à une époque où les savoirs occidentaux jouent un rôle important mais controversé, ils sont essentiellement basés sur les savoirs chinois; ceux-ci sont donc à la base de sa formation. En mathématiques, il connaît les ouvrages de Ricci, qui remontent au début du XVII ${ }^{\mathrm{e}}$ siècle; il ne semble pas avoir accès à des ouvrages plus récents. Deux raisons peuvent expliquer que ceux-ci, notamment les Livres calendaires suivant la nouvelle méthode occidentale, soient plus difficilement accessibles. La plupart des ouvrages de cette collection ont été imprimés pour la première fois dans les années 1630, peu de temps avant la chute des Ming. D'autre part, les ouvrages jésuites, alors produits pour l'institution impériale, n'ont peut-être pas circulé autant que des textes écrits antérieurement comme la traduction des Éléments, qui était précisément destinée à des lettrés comme Mei Wending.

\section{La capitale du Sud}

Le district de Xuancheng fut rattaché à la province de l'Anhui en 1667, lorsque la province du Jiangnan fut divisée en deux : Jiangsu et Anhui. Scinder

17. Li Yan 1998: 521.

18. Wu'an lisuan shuji. SKQS 795: 965-966; le district de Xuancheng faisait partie de la préfecture de Ningguo, et abritait le siège de l'administration préfectorale. 
le Jiangnan semble avoir fait partie d'une politique visant à affaiblir Nankin (Nanjing, littéralement «capitale du Sud»), l'ancienne capitale secondaire des Ming; celle-ci est déchue de son statut et rebaptisée Jiangning par les Qing. Elle n'en reste pas moins la capitale du Sud - le Sud désignant en chinois la région du Jiangnan - sur les plans culturel et intellectuel. Malgré la partition en deux provinces, Nankin est restée le siège de l'examen provincial qui avait lieu à l'automne une fois tous les trois ans pour les candidats de l'Anhui comme pour ceux du Jiangsu ${ }^{19}$. À l'occasion de ces examens, un grand nombre de lettrés convergeaient donc vers la ville; c'était l'occasion pour eux non seulement de se rencontrer et d'échanger des informations et des idées, mais aussi de consulter ou d'acquérir des livres. Ce n'est pas un hasard si le premier séjour de Mei à Nankin dont nous avons connaissance remonte à l'automne 1666, au moment d'une session triennale de l'examen provincial. Rien n'indique s'il s'est alors présenté à l'examen. Il nous dit en revanche que «[...] à la librairie de $\mathrm{M}$. Yu, [il s'est] procuré un ouvrage imprimé, à l'intérieur duquel il y a des cartes célestes [...]». En évoquant cet achat, Mei n'indique pas s'il s'agit d'un des ouvrages des jésuites ${ }^{20}$. Il semble qu'il s'est à nouveau rendu à Nankin en 1669, année où avait lieu la session suivante de l'examen provincial. C'est alors qu'il aurait rencontré pour la première fois le fils cadet du grand savant Fang Yizhi (1611-1671), Fang Zhongtong (1634-1698), dont Mei cite d'ailleurs l'opinion dans sa Discussion sur les tableaux rectangulaires ${ }^{21}$. Fang Zhongtong est lui-même l'auteur d'un traité mathématique, l'Expansion des nombres et de la mesure (Shudu yan). Achevé en 1661 et imprimé en 1687, celui-ci se présente comme une anthologie des connaissances mathématiques de son auteur ${ }^{22}$. Peu après cette rencontre avec le fils, Mei entre en correspondance avec le père, qui vivait alors retiré dans un temple bouddhiste du Jiangxi ${ }^{23}$.

L'un des sujets de conversation entre Mei Wending et Fang Zhongtong est un instrument de calcul que ni l'un ni l'autre n'ont encore vu: les réglettes de Neper (chousuan), introduites en Chine par les jésuites dans les années $1630^{24}$. Il s'agit d'un ensemble de réglettes portant chacune une table de multiplication et qui servent d'auxiliaire pour effectuer cette opération (figure 1). Un manuel,

19. Magone $2001: 63$.

20. Mei Wending $1995: 141$.

21. Li Di $2006: 83$.

22. Jami 2012: 44-49.

23. Li Yan 1998: 520.

24. Cervera 2011. 
intitulé Chousuan, inclus dans les Livres du calendrier de l'ère Chongzhen, les décrit et en explique l'usage.
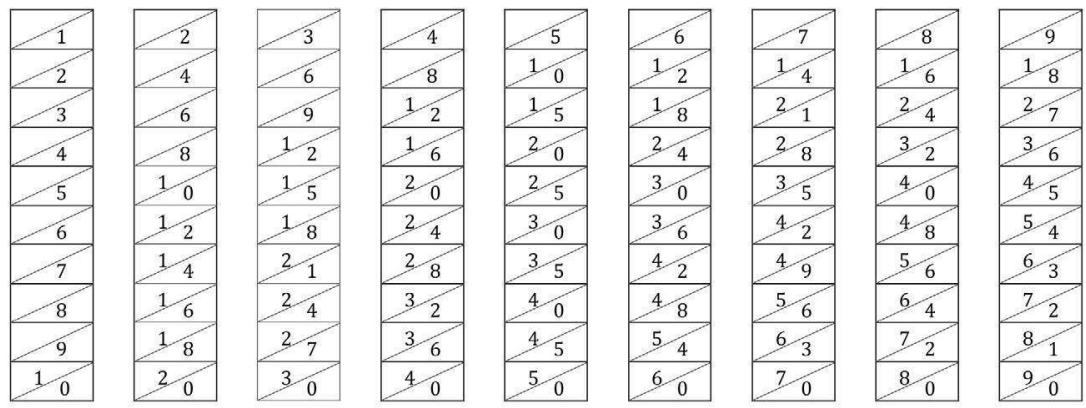

\section{$2 / 4 \longdiv { 3 } 2$}

Figure 1

Les réglettes de Neper représentent les tables de multiplication. Elles servent d'auxiliaire à la multiplication (et à la division). Ainsi pour multiplier un nombre par 34, on place les réglettes 3 et 4 l'une contre l'autre. On obtient les multiples de 34 sur chaque ligne en additionnant les deux chiffres du milieu. Ainsi 8 x 34 est calculé en combinant 24 et 32 : les trois chiffres du produit seront $2,4+3$ et 2 ; ce produit est donc 272 .

Peu après sa conversation avec Fang, Mei a pour la première fois entre les mains un jeu de réglettes de Neper que l'un de ses parents a rapporté de la capitale $^{25}$, sans avoir connaissance du manuel des jésuites qui en explique l'usage. Mei rédige alors lui-même un traité sur le sujet, qu'il intitule Les Réglettes de Wu'an (Wu'an chousuan; Wu'an était son surnom - zi). Achevé en 1678, il est imprimé à Nankin en 1681; ce serait donc le premier de ses ouvrages à être imprimé ${ }^{26}$. Ainsi les instruments, moins faciles à reproduire que les livres, étaient-ils aussi des véhicules des savoirs mathématiques et astronomiques. Comme les livres, ils pouvaient faire l'objet d'une mise par écrit en guise d'appropriation: comprendre, interpréter un livre donne lieu à un commentaire; les instruments, eux, nécessitent un mode d'emploi. La circulation des instruments s'accompagne parfois d'adaptations: ainsi Mei

25. Li Yan 1998: 520 .

26. Li Di $2006: 84,145$; Li Yan 1998: 526. 
décide-t-il de changer l'orientation des réglettes de Neper, en accord avec son choix d'écrire les nombres verticalement, comme on écrit les textes en chinois, et non plus horizontalement comme le faisaient les Européens pour poser les calculs (figure 2).
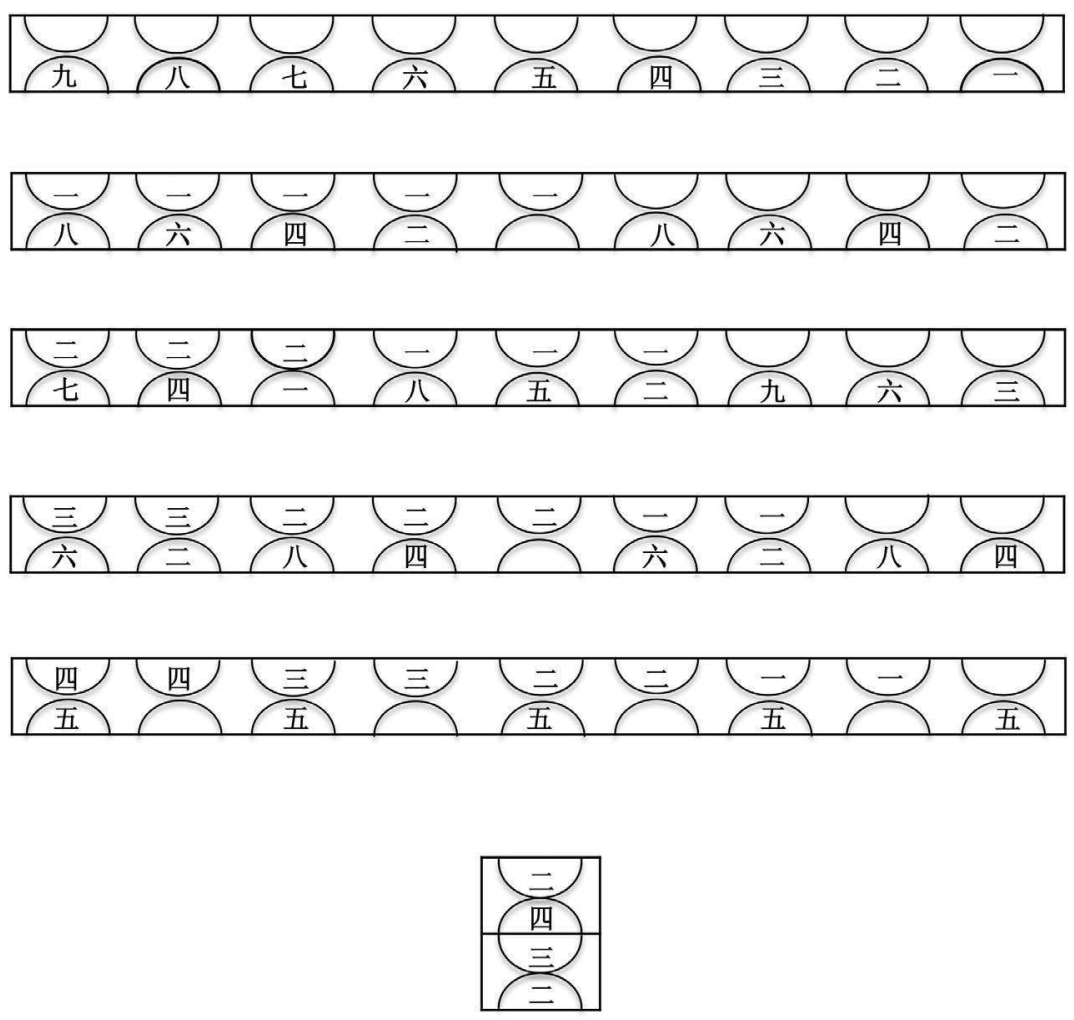

Figure 2

Les cinq premières réglettes redessinées par Mei Wending. Les nombres sont écrits verticalement et disposés horizontalement de droite à gauche. Lorsqu'on place deux réglettes l'une au-dessus de l'autre, les deux chiffres à additionner sont dans un cercle. Pour effectuer 8 x 34, on lit ainsi les trois chiffres du produit l'un sous l'autre: 2, 4+3 et 2 ; ce produit est donc 272 .

Cet instrument est particulièrement important dans l'arithmétique écrite élaborée par Mei: pour la multiplication, il adopte en effet la méthode dite 
«par jalousie », qu' on rencontre déjà en Chine avant l'arrivée des jésuites, sous le nom de pudijin. Il s'agit de disposer les nombres à multiplier l'un en ligne, l'autre en colonne, au-dessus et sur le côté droit d'un tableau à double entrée. On effectue chaque multiplication élémentaire dans la case correspondante, pour ensuite additionner les résultats partiels comme on le fait avec les réglettes de Neper ${ }^{27}$.

Mei se présente de nouveau à l'examen provincial en 1672, puis en 1675 , toujours sans succès. Il passe cinq ans à Nankin à partir de cette dernière date. Il y est donc présent lors de la session suivante de l'examen, en 1678; il est probable qu'il tente alors une dernière fois sa chance ${ }^{28}$. On peut d'ailleurs s'interroger sur son désir de mener une carrière de fonctionnaire: plus tard dans sa vie, il sera critique vis-à-vis du système des examens impériaux ${ }^{29}$. Cependant, en tant qu'aîné d'une fratrie de quatre enfants, il était sans doute attendu de lui qu'il rehausse le prestige et le statut de la famille Mei. Quoi qu'il en soit, ce qu'on sait de ses visites puis de son long séjour à Nankin suggère qu'il y a consacré plus de temps à lire des traités de mathématiques et d'astronomie que des commentaires sur les classiques ou des manuels de préparation aux examens. L'accès à ces traités spécialisés lui est certes rendu possible par le marché du livre à Nankin, mais aussi grâce aux relations que Mei entretient avec certains lettrés qui résident dans la ville. Ainsi, l'exemplaire des Livres calendaires de l'ère Chongzhen qu'il a acheté à Nankin dès 1675 s'avère incomplet: il y manque l'Explication du compas de proportion (Bili

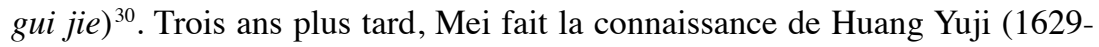
1691), bibliophile renommé, dont la collection comportait 60000 juan $^{31}$. Huang prête son exemplaire de l'ouvrage manquant à Mei; celui-ci le recopie pour compléter le compendium impérial d'astronomie ${ }^{32}$. C'est également dans la bibliothèque de Huang Yuji que Mei prend connaissance du premier des Neuf chapitres sur les procédures mathématiques (Jiuzhang suanshu, $\mathrm{I}^{\mathrm{er}}$ siècle de l'ère commune), le classique mathématique qui est alors perdu ${ }^{33}$.

Plus généralement, il ressort que l'accès aux livres spécialisés qui intéressent Mei Wending doit autant sinon davantage aux prêts entre lettrés qu'au marché de l'imprimé. Ses ouvrages regorgent de notes indiquant qu'il

27. Jami 2012: 88-90.

28. Li Di 2006 : 427; Li Yan 1998: 520.

29. Mei 1995 : 97 ; cité par Elman $2000: 528$.

30. Li Yan 1998: 522.

31. McDermott $2006: 160$.

32. Li Yan 1998: 523.

33. Sur cet ouvrage, voir Chemla \& Guo 2004. 
a copié l'exemplaire appartenant à tel ou tel lettré d'un livre d'astronomie ou de mathématiques, pratique qu'il continuera toute sa vie. Un voyage au Fujian, effectué alors qu'il était dans sa soixante-septième année, semble ainsi avoir eu pour but de copier un traité qu'il n'avait pas encore pu lire ${ }^{34}$. Les sources nous donnent donc à voir la mobilité de Mei et même sa sociabilité comme largement motivées par sa quête des savoirs mathématiques et astronomiques. Par ailleurs, il semble qu'il faisait lui-même la copie. Or copier un texte pour se l'approprier n'est pas forcément un pis-aller; on aurait plutôt là un aperçu de sa pratique de savant.

Pendant les années qu'il passe à Nankin, c'est à Ma Decheng, un savant musulman versé en astronomie, avec lequel il s'est lié d'amitié, que Mei doit d'avoir pris connaissance d'un grand nombre de traités d'astronomie; Ma l'autorise à recopier les exemplaires en sa possession. La présence à Nankin d'un savant musulman versé en astronomie n'a rien de surprenant. En effet, au début de la dynastie Ming, à l'époque où la ville était encore capitale, un travail de traduction d'ouvrages d'astronomie «musulmane» avait été commandité par l'empereur Hongwu (r. 1368-1398). Le Bureau de l'astronomie comportait alors une «Section musulmane» (Huihui ke); celle-ci existait toujours au XVII ${ }^{\mathrm{e}}$ siècle, et fut fermée en 1657 à la suite des conflits qui opposèrent ses membres à Adam Schall ${ }^{35}$. Sous les Ming, les savoirs astronomiques se transmettaient souvent dans les familles d'une génération à l'autre. Ma Decheng était sans doute un descendant de l'un des astronomes musulmans qui s'étaient fixés à Nankin à la fin du XIV $v^{e}$ siècle. Il n'est donc guère surprenant qu'il ait notamment fait connaître à Mei les ouvrages «musulmans » compilés au début des Ming, mais aussi des observations faites en dehors des institutions impériales. En 1680, à sa demande, Mei corrige une table des longueurs de l'ombre d'un gnomon d'une hauteur de 10 chi (environ 3 mètres) aux jours des vingt-quatre $q i$ (instants divisant l'année tropique en vingt-quatre parties égales). Cette table était basée sur des observations faites dans les mosquées de quatre provinces $\left(\right.$ Shaanxi, Henan, Bei Zhili et Jiangnan) ${ }^{36}$. Grâce à Ma Decheng, Mei Wending a donc découvert non seulement une tradition textuelle, mais aussi un réseau d'observation astronomique couvrant plusieurs provinces et indépendant de l'État impérial. C'est l'implantation géographique des musulmans dans les provinces centrales du territoire impérial des Ming qui dessine la carte des observations communiquées à Mei Wending.

34. Li Yan 1998: 536.

35. Jami 2012: 40-41.

36. SKQS 795: 969. 
Mais leurs échanges ne sont pas limités à l'astronomie musulmane: Mei Wending doit encore à Ma Decheng d'avoir eu connaissance de la publication de la Véritable origine des mouvements célestes (Tianbu zhenyuan, 1653), ouvrage du jésuite Nikolaus Smogulecki (1610-1656). Celui-ci avait résidé à Nankin de 1651 à 1653, où il avait enseigné les mathématiques et l'astronomie ; il y avait laissé la réputation d'un homme qui ne cherchait pas à convertir ceux qui venaient vers lui pour parler de sciences mathématiques ${ }^{37}$. Parmi les savants chinois ayant étudié auprès de lui se trouvaient non seulement Fang Zhongtong, mais aussi Xue Fengzuo (1600-1680), dont Mei Wending découvre également l'ouvrage Intégration des études astronomiques (Lixue huitong, 1664) grâce à Ma Decheng. Xue avait collaboré avec Smogulecki sur la Véritable origine des mouvements célestes, dont il utilisa certains passages pour critiquer l'astronomie des jésuites du Bureau de l'astronomie ${ }^{38}$. En somme, on pourrait dire qu'à Pékin il y avait deux camps en astronomie: les jésuites et leurs ennemis - parmi lesquels les astronomes musulmans du Bureau. À Nankin la situation était plus complexe: c'est grâce à un savant musulman que Mei Wending prit connaissance d'un volet des «savoirs occidentaux» qui, bien qu'étant le fruit de l'enseignement d'un missionnaire jésuite, présentait des différences significatives avec ce qu'on appelle souvent «l'astronomie jésuite en Chine». Un poème composé par Mei en hommage à Xue souligne que ce dernier, «bien que n'ayant jamais servi Jésus, a exploré toutes les techniques» des missionnaires en matière d'astronomi ${ }^{39}$. À Nankin, la circulation des savoirs n'était donc pas tributaire des appartenances religieuses. Même si, on l'a vu, il fallait parfois un certain temps pour que les livres et instruments «occidentaux» produits à Pékin arrivent à Nankin, cette dernière n'en était pas moins un lieu de savoir cosmopolite: loin des institutions impériales où se déroulaient des conflits aigus entre méthodes chinoises et occidentales, il semble avoir été possible de s'approprier l'ensemble des savoirs de l'astronomie sans choisir son camp.

Les écrits de Mei Wending pendant son long séjour à Nankin et pendant les années qui suivent ce séjour reflètent l'élargissement considérable des informations auxquelles il a accès. Il continue à rédiger des commentaires sur les textes qu'il lit, comme il le faisait à Xuancheng. Ainsi, il consacre deux commentaires (buzhu) aux livres d'astronomie «musulmane». Mais plus généralement, il lit désormais textes «chinois», «musulmans» et «occiden-

37. Engelfriet 1998a; Jami 2012: 43.

38. Shi 2007 : en particulier 87-92.

39. Mei $1995: 239$. 
taux » à la lumière les uns des autres, chaque tradition textuelle, chaque auteur particulier lui permettant de vérifier, corriger ou critiquer les autres.

Mei Wending semble avoir fait assez peu d'observations. Cependant il s'intéressait aux instruments, astronomiques comme mathématiques, et même à diverses machines et dispositifs techniques ${ }^{40}$. On sait qu'il a dessiné des cadrans solaires ${ }^{41}$. Si l'on ignore de quels moyens il disposait pour la fabrication, ses écrits donnent des descriptions détaillées des améliorations qu'il propose d'apporter à certains instruments. Cependant, sur la dizaine de titres de ce genre (consistant chacun en un seul juan), aucun n'a été inclus dans les collections qui regroupent ses œuvres ${ }^{42}$. En revanche, ses écrits sur les instruments de calcul ont été publiés: outre les réglettes de Neper, il a écrit sur le compas de proportion, sur le calcul écrit - qu'il propose de substituer à l'abaque pour les quatre opérations élémentaires de l'arithmétique -, et sur les baguettes à calculer de la Chine ancienne. Il est intéressant de noter que ces traités qui présentent les diverses méthodes de calcul numérique, et qui sont beaucoup plus élémentaires que le Traité sur les tableaux rectangulaires, ont semble-t-il été écrits pendant le séjour à Nankin. On peut penser que ses échanges avec des lettrés moins versés que lui en mathématiques l'ont amené à écrire à leur intention des initiations aux savoirs occidentaux en mathématiques. Ici, deux points sont à souligner: en adoptant comme base $\mathrm{du}$ calcul une technique utilisant pour tout instrument les «quatre trésors du cabinet de travail» (wenfang sibao: papier, pinceau, encre et encrier), Mei intègre les mathématiques aux savoirs lettrés. D'autre part, il adopte ici la posture d'un maître, alors que sa renommée s'étend dans les milieux lettrés: il espère jouer ce rôle auprès de certains d'entre eux.

Les deux traités de mathématiques mentionnés plus haut font partie des neuf titres que Mei voulait faire imprimer dans une collection au titre programmatique: Intégration des mathématiques chinoises et occidentales (Zhongxi suanxue tong). En 1680, un fascicule de préfaces et d'avant-propos de cette collection est imprimé; il semble que le projet d'édition s'arrête là. Cela reflète sans doute l'intérêt assez faible des lettrés pour les sciences mathématiques. En revanche, deux ans après son achèvement en 1672, le Traité sur les tableaux rectangulaires avait été recopié par un certain nombre de savants, dont faisaient partie Fang Zhongtong et Huang Yuji ; un passage de l'ouvrage mentionne d'ailleurs une divergence de vue entre Mei et Fang quant

40. Tian et Zhang 2006.

41. Zhu Yizun, Pushuting ji, SKQS $1318: 334$.

42. SKQS 795: 979-981; Li Di 2006 : 123-127. 
aux fondements des mathématiques ${ }^{43}$. Premier ouvrage de Mei à circuler, le Traité sur les tableaux rectangulaires est le second à être imprimé, en 1687 , par les soins de Li Dingzheng, non pas au Jiangnan, mais à Quanzhou (Fujian) dont Li était originaire. Quanzhou est situé à environ mille kilomètres de Nankin, et Li Dingzheng était à l'époque magistrat du district de Jiayu, au Hubei, situé à plus de huit cents kilomètres en amont de Nankin sur le Fleuve Yangzi : cela nous donne une idée de la portée de la circulation d'un ouvrage aussi spécialisé sous forme de manuscrit ${ }^{44}$. Il n'est pas surprenant que cette portée soit aussi celle de la mobilité géographique des fonctionnaires de l'État impérial.

L'intégration de Mei aux cercles savants de Nankin se traduit aussi par la demande qui lui est faite de rédiger le chapitre consacré à la «répartition des domaines » pour la Monographie provinciale du Jiangnan (Jiangnan tongzhi, 1684). Le changement d'échelle par rapport au chapitre qu'il avait rédigé pour la Monographie locale de Ningguo, qui concernait une simple préfecture, marque bien l'étendue de sa renommée à la région. Autre indication de cette renommée, dans les années qui suivent son retour de Nankin il reçoit chez lui la visite d'un certain nombre de savants ${ }^{45}$. Il voyage également: il passe ainsi l'hiver 1688 à Hangzhou. Parmi les personnes qu'il y rencontre pour discuter de mathématiques et d'astronomie, on compte le missionnaire jésuite italien Prospero Intorcetta (1626-1696); celui-ci n'est d'ailleurs pas connu comme particulièrement versé en sciences mathématiques; il aurait cependant participé à l'observation d'une éclipse de soleil qui s'est produite en avril de cette année-là, également observée par d'autres jésuites dans d'autres villes de Chine $^{46}$. Il semblerait qu'aucun missionnaire jésuite versé dans ces sciences n'ait résidé à Nankin durant les années où Mei y a séjourné ${ }^{47}$; ce dernier aurait cherché à rencontrer l'un de ceux qui étaient relativement proches de lui géographiquement.

43. Tong et Feng $2007: 720-721$; Jami 2012: 92.

44. Li Yan 1998: 528, Li Di $2006: 431$.

45. Li Di $2006: 431$.

46. Lecomte $1990: 511-512$.

47. Li Yan 1998: 528-529; The Ricci Roundtable, http://ricci.rt.usfca.edu/index.html (consultée le 1er mai 2013). 


\section{Pékin, capitale des Qing}

En 1678, trois ans après l'installation de Mei Wending à Nankin, l'empereur Kangxi décide la tenue d'un examen extraordinaire, dans le but de recruter des savants pour travailler à la compilation de l'histoire officielle de la dynastie Ming $^{48}$.Il suit ainsi la tradition impériale chinoise, qui veut que chaque nouvelle dynastie écrive l'histoire de celle qui l'a précédée sur la base des annales dont elle a hérité; il s'efforce aussi d'attirer au service de ce projet certains grands lettrés qui se sont jusque-là abstenus de servir les Mandchous par loyauté envers la dynastie Ming. Shi Runzhang, qui avait dirigé la Monographie de Xuancheng, et Pan Lei, qui avait écrit en 1672 une préface à la Discussion des tableaux rectangulaires, sont tous deux lauréats de cet examen. Lorsque le travail de compilation commence l'année suivante, Shi écrit à Mei pour lui proposer de venir à Pékin travailler au chapitre de l'Histoire des Ming (Mingshi) qui doit, suivant l'usage, être consacré au système calendaire des Ming. Mei décline l'invitation, arguant qu'il ne peut se soustraire à des engagements qu'il a pris. Demeurant à Nankin, il rédige cependant une première ébauche sur le sujet, qu'il mentionne dans sa bibliographie sous le titre Paroles superflues à propos de la monographie sur l'astronomie (Lizhi zhuiyan), et l'envoie à $\mathrm{Shi}^{49}$. Une dizaine d'années plus tard, en 1689, Mei se rend à Pékin. Ce voyage pourrait être lié à la compilation de l'Histoire des Ming, puisque peu après son arrivée, il rédige un Projet de monographie sur l'astronomie pour l'Histoire des Ming (Mingshi lizhi nigao). Il a alors eu entre les mains une autre ébauche de cette monographie due au grand savant Huang Zongxi (1610-1695), qui était assez versé dans les sciences mathématiques; ce dernier avait rédigé cette ébauche dans le Sud et n'était pas à la capitale. Mei dit avoir trouvé plus de cinquante erreurs dans cette ébauche. Il considère par ailleurs les savants de l'Office de l'Histoire des Ming (Mingshi guan) comme peu compétents dans le domaine de l'astronomie ${ }^{50}$.

Peu après son arrivée, Mei devient l'hôte du frère aîné de Li Dingzheng, Li Guangdi (1642-1718), alors Chancelier de l'académie Hanlin (zhang yuan xueshi). Reçu à l'examen métropolitain dès 1670, représentant éminent de l'école philosophique de Cheng Yi et Zhu Xi, Li Guangdi fait partie de ceux qui ont enseigné les classiques à l'empereur. Si le souverain mandchou reconnaît l'autorité des savants chinois dans ce domaine, il affiche en revanche une piètre estime pour leur connaissance des sciences mathématiques, et se pose

48. Wilhelm 1951.

49. SKQS 795: 966.

50. Jami 2012: 214-215. 
ainsi à son tour en maître vis-à-vis d'eux. C'est certainement pour lui plaire que Li Guangdi s'est mis à l'étude de ces sciences. Les ayant d'abord étudiées avec Pan Lei, de l'enseignement duquel il est peu satisfait, Li invite Mei à devenir son professeur. Ils travaillent notamment sur les Réglettes de Wu'an ${ }^{51}$. Dans le même temps, Li Guangdi incite Mei à abandonner son projet d'un grand traité d'astronomie en cinquante-huit chapitres, au profit d'un ouvrage concis qui soit à la portée de lettrés non spécialistes comme Li lui-même. Sous l'étroite surveillance de ce dernier, Mei commence donc en 1691 à rédiger un ouvrage en forme de dialogue, dans lequel un savant versé dans les sciences mathématiques, qui pourrait bien être Mei, répond aux Doutes sur l'astronomie (Lixue yiwen) d'un autre savant aussi érudit et orthodoxe qu'ignorant de ce sujet technique, qui pourrait bien être Li Guangdi ${ }^{52}$. Cet ouvrage présente les similitudes et les différences entre les systèmes chinois (zhong), occidental (xi), et musulman (huihui). Mei y défend l'idée que l'astronomie, qui a été confiée à des spécialistes par les empereurs de la haute Antiquité, progresse au cours de l'histoire et que certaines notions introduites par les jésuites, comme la sphéricité de la Terre, ne sont pas en contradiction avec la cosmologie chinoise traditionnelle. Plus encore, il affirme que l'astronomie introduite par les jésuites est en fait d'origine chinoise ${ }^{53}$.

À Pékin comme à Nankin, Mei rencontre un certain nombre de grands lettrés qui le reconnaissent comme un expert dans les domaines des sciences mathématiques ${ }^{54}$; son nom est mentionné dans les recueils de prose (wenji) de plusieurs d'entre eux; certains donnent des préfaces à ses ouvrages. Contrairement à ceux qu'il a connus à Nankin, ces grands lettrés sont aussi des hauts fonctionnaires. Il faut souligner que les deux milieux ne sont pas disjoints: c'est Shi Runzhang, que Mei a connu pendant une période où le premier s'était retiré chez lui, qui, en partant à Pékin, a sollicité la contribution de Mei à l'Histoire des Ming. Pan Lei est lui aussi à Pékin pour y travailler. Et l'on peut penser que Li Guangdi avait entendu parler de Mei Wending par son frère Dingzheng, qui avait fait imprimer la Discussion sur les tableaux rectangulaires.

À Pékin, Mei espérait rencontrer Ferdinand Verbiest ${ }^{55}$; mais ce dernier était mort en 1688. Si l'expertise des jésuites était ce qui avait attiré Mei à la capitale, son séjour, qui dura cinq ans, ne fut pas un succès à cet égard.

51. Jami 2012: 215-216.

52. Jami 2012: 216-220.

53. Jami $2004: 717-725$.

54. Li Yan 1998: 530.

55. Li Yan 1998: 529. 
Il rencontra au moins une fois Antoine Thomas (1644-1709), à qui la tâche d'astronome impérial était échue à la mort de Verbiest. Mei a noté qu'une conversation eut lieu entre eux le 14 janvier 1691 - ce fut peut-être la seule -. Thomas lui dit alors qu'il existait une méthode différente de celle utilisée par les fonctionnaires qui calculaient les impôts pour trouver l'aire d'un champ. Mei, d'abord en désaccord avec cette idée, y réfléchit par la suite, et mit au point une «méthode pour mesurer une aire plane à distance» (yao liang pingmian $f a)^{56}$. Il n'y a à ma connaissance aucune autre mention d'une discussion portant sur les sciences mathématiques entre Mei et les jésuites. Certes, les jésuites de Pékin, qui comme Thomas travaillaient non seulement au Bureau de l'astronomie mais aussi à la Maison impériale, n'étaient pas intégrés aux milieux des lettrés fonctionnaires comme l'avait été Matteo Ricci. Mais cela ne suffit pas à expliquer pourquoi les sources ne mentionnent aucun échange entre les deux personnages qui apparaissent aujourd'hui comme les plus versés en sciences mathématiques alors présents dans la capitale, et dont l'un y était venu tout exprès. L'explication se trouve du côté de la cour impériale.

En 1690, Kangxi retourne à l'étude des mathématiques, qu'il avait abordée avec Verbiest dans les années 1670, sous la tutelle de nouveaux professeurs jésuites qui rédigent pour lui plusieurs ouvrages. Antoine Thomas lui enseigne le calcul, tandis que deux jésuites français traduisent pour lui, d'abord en mandchou puis en chinois, un nouveau manuel de géométrie qui doit remplacer les Éléments d'Euclide traduits par Ricci. Ce travail de rédaction et de traduction se fait dans le plus grand secret, à la demande expresse de Kangxi qui entend garder le monopole des nouveaux savoirs occidentaux ainsi mis à sa disposition ${ }^{57}$. Aucun lettré chinois ne doit lui disputer le rôle de maître en mathématiques qu'il s'est lui-même attribué. Il est significatif à cet égard que l'empereur ait alors acquis une piètre opinion de Mei Wending, comme il le fait savoir aux hauts fonctionnaires qui l'entourent alors que Mei est toujours à Pékin, en 1692:

Ces temps derniers il y a un homme du clan Mei du Jiangnan; nous avons entendu dire qu'il maitrise parfaitement les mathématiques. Nous avons envoyé quelqu'un pour le tester: ce qu'il dit sur la mesure de l'ombre du gnomon ne correspond pas [à la réalité]. [...] Sa méthode de calcul n'étant pas exacte, il utilise un gnomon court pour mesurer les ombres, pour que sa tromperie ne soit pas visible ${ }^{58}$.

56. Jami 2012: 216-217.

57. Jami 2012: chap. 8 et 9.

58. Zhang Yushu, Zhang Wenzhen ji. SKQS 1322: 389-687, 518; cité par Jami 2012: 231. 
À ma connaissance, ni les écrits de Mei ni ceux de Li Guangdi ne mentionnent ce jugement cinglant, dont on peut se demander s'il est impartial. Quoi qu'il en soit, il faut reconnaitre que l'empereur comprend l'importance de la précision dans les mesures; c'est aussi pour lui un terrain sûr, car aucun savant n'a des moyens comparables aux siens pour la fabrication, l'entretien et l'utilisation des instruments astronomiques. De plus, la précision des mesures est vitale pour l'astronomie impériale: elle seule permet de déterminer le moment exact où doivent être conduits certains rituels. L'enjeu est ni plus ni moins que la préservation de l'harmonie entre les rythmes du monde humain et ceux du cosmos, dont l'empereur est garant. Deuxième empereur mandchou à régner à Pékin, Kangxi s'avère particulièrement soucieux d'assumer à la lettre la responsabilité cosmologique qui est la sienne s'il veut être un empereur dans la tradition chinoise, et pas seulement un conquérant mandchou.

Tout ceci donne à penser que ce n'est pas un hasard si Li étudie avec Mei et lui fait rédiger les Doutes sur l'astronomie au moment même où Kangxi fait rédiger des manuels de mathématiques par les jésuites. En prenant Mei sous sa protection, Li Guangdi cherche moins à satisfaire son propre goût pour les sciences mathématiques que celui de l'empereur. Il s'agit de donner à voir à l'empereur une expertise mathématique émanant de la communauté lettrée qui fasse pièce à celles des jésuites, et de mettre cette expertise à sa disposition. Plus que sur la compétence de Mei Wending, ou sur celle de l'empereur luimême, le rejet du premier par le second en dit long sur la spécificité des critères de l'astronomie impériale et sur les enjeux politiques et culturels que Kangxi a placé dans les sciences mathématiques.

En 1693, Mei quitte la capitale pour rentrer chez lui ${ }^{59}$. S'il n'a pas gagné la faveur de l'empereur, les cinq années passées dans la capitale ont tout de même été doublement fécondes: il a composé plusieurs traités, qui sont dotés de préfaces rédigées par de prestigieux savants et hauts fonctionnaires. $\mathrm{Li}$ Guangdi rédige lui-même une préface aux Doutes sur l'astronomie qu'il fait imprimer en 1696, alors que son auteur voudrait encore le réviser et y ajouter des cartes ${ }^{60}$. Cet ouvrage, s'il ne satisfait pas entièrement Mei, lui ouvrira finalement la voie de la consécration.

59. Li Di $2006: 433$.

60. Mei $1995: 22-23$; Chu $1994: 186$. 


\section{Chez le gouverneur du Zhili}

En 1699, Li Guangdi est nommé gouverneur du Zhili (actuel Hebei), dont la capitale se trouvait à Baoding. Vers la fin 1702, au cours d'une audience avec l'empereur, Li lui offre un exemplaire des Doutes sur l'astronomie. Quelques mois plus tard, Kangxi lui offre en retour deux ouvrages rédigés par ses professeurs jésuites dans les années 1690 pour leurs cours de mathématiques: les Éléments de géométrie (Jihe yuanben) et les Éléments de calcul (Suanfa yuanben). Le premier est une traduction partielle du manuel de géométrie d'Ignace Gaston Pardies (1636-1673), qui a été professeur de mathématiques au Collège jésuite de Paris ${ }^{61}$. Le second est basé sur le livre VII des Éléments d'Euclide, avec quelques propositions du livre VIII et la première proposition du livre IX: il s'agit des livres d'arithmétique. Dans la conception duale des mathématiques qui est celle des jésuites, les deux traités en chinois forment un tout. Les Éléments de calcul, qui donnent les fondements de l'arithmétique, semblent avoir été conçus comme le pendant des Éléments de géométrie ${ }^{62}$. L'échange de livres, tel que celui entre Li Guangdi et l'empereur Kangxi, avait cours parmi les bibliophiles de l'époque; il était parfois régi par des contrats ${ }^{63}$. Cependant, il s'agit ici d'ouvrages qui ont été écrits sur commande - les Doutes sur l'astronomie pour Li Guangdi, les Éléments de géométrie et les Éléments de calcul pour l'empereur. Ainsi deux mécènes des sciences mathématiques partagent les fruits de leur patronage. L'opinion de l'empereur sur les Doutes sur l'astronomie est mitigée; tout en reconnaissant les années de travail qui ont rendu possible l'écriture du livre, il déplore l'absence de calculs ${ }^{64}$. On assiste pourtant à un changement d'attitude de sa part, en comparaison avec la décennie précédente où il s'était assuré le monopole de l'enseignement des jésuites. De toute évidence, l'échange n'est pas symétrique: les sources indiquent que, tandis que l'empereur lit le texte de Mei pour le corriger, $\mathrm{Li}$ s'efforce de comprendre les deux manuels qui lui ont été offerts en retour. Les formes qui font de l'empereur le maître et de tous les Chinois ses élèves sont ainsi respectées. C'est "parce qu'il ne les comprend pas tout à fait que [Li Guangdi] invite Mei Wending au siège [de son administration provinciale] pour en discuter avec lui à ses heures de $\operatorname{loisir}^{65}{ } »$.

61. Pardies 1671; voir Jami 2012: 160-179.

62. Jami 2012: 195-200.

63. McDermott $2006: 155-163$.

64. Jami 2012: 247-248.

65. Li Yan 1998: 539-540. 
Ce dernier est alors dans sa soixante-douzième année; toujours aussi prolifique, il a plus de quatre-vingts titres à son actif ${ }^{66}$. Il accepte l'invitation, et se rend à Baoding en compagnie de son frère Wenmi, de son fils Yiyan (1655-1705) et de l'aîné de ses petits-fils Mei Juecheng (1681-1763): ce sont ainsi trois générations de sa famille versées dans les sciences mathématiques qui l'accompagnent. Li Guangdi, de son côté, rassemble des élèves pour ce maître parmi ses jeunes protégés: Wei Tingzhen (1669-1756), Wang Lansheng (1679-1737), Chen Wance (1667-1734), Wu Yongxi (dates inconnues), et son fils aîné Li Zhonglun (1663-1706) ${ }^{67}$. Il entreprend ainsi de faire former aux mathématiques une nouvelle génération, à la disposition de laquelle il met à la fois l'expertise de Mei et ce que l'empereur a bien voulu partager avec lui de ses «nouvelles mathématiques». Mais Li reproduit aussi dans sa résidence de gouverneur le modèle institué par l'empereur au Palais impérial, à savoir un enseignement de mathématiques et d'astronomie: Kangxi en faisait dispenser un à ses fils par Antoine Thomas ${ }^{68}$. Les sujets abordés à Baoding ne sont pas limités aux deux manuels impériaux; ainsi, une discussion sur le système calendaire des Yuan amène Mei à rédiger une Explication détaillée du calcul des anomalies moyenne, cubique et apparente (Ping li ding sancha xiangshuo) dans ce système ${ }^{69}$.

Pendant le séjour de Mei à Baoding, Li Guangdi continue à promouvoir son protégé. Il fait imprimer plusieurs de ses ouvrages, dont un Résumé de la trigonométrie (Sanjiaofa juyao). Il trouve également l'occasion de le recommander à l'empereur, si bien que lorsque ce dernier revient de l'une de ses tournées d'inspection dans le Sud (nanxun) au printemps 1705, Mei Wending est reçu en audience sur le bateau impérial lors d'une escale de celuici. La discussion se prolonge tard dans la nuit et se poursuit le lendemain. Le surlendemain, le bateau impérial continue son chemin, et Mei va saluer et remercier l'empereur à l'étape suivante. Nous avons quelques indications sur la teneur de la longue conversation entre les deux hommes. Mei Wending offre à l'empereur un exemplaire de son Résumé de la trigonométrie. Situé de plain pied dans le champ des savoirs occidentaux, celui-ci part de définitions élémentaires, puis mène le lecteur des triangles vers les polygones inscrits dans un cercle ou circonscrits à un cercle, pour se clore sur un chapitre consacré aux mesures à distance. L'ouvrage est beaucoup plus technique que les Doutes sur

66. Li Yan 1998: 536, cite les chiffres de cinquante-huit titres d'astronomie et de vingtdeux titres de mathématiques pour 1699.

67. Li Di 2006 : 436; Jami 2012: 247.

68. Jami 2012: 244-245.

69. SKQS 795: 982. 
l'astronomie, et est certainement plus au goût de l'empereur. Mais surtout, il vient à point nommé : quelques mois plus tôt, l'empereur a dicté et fait imprimer en chinois et en mandchou une courte Discussion sur les triangles et le calcul (Sanjiaoxing tuisuanfa lun). Faute de connaître la date exacte à laquelle Li Guangdi a fait graver les planches du Résumé de la trigonométrie pour son impression, on est tenté de supposer qu'après avoir pris connaissance de l'essai impérial, il a voulu mettre en avant un ouvrage dont le contenu fait écho au titre choisi par Kangxi. La Discussion sur les triangles et le calcul donne le point de vue impérial sur les sciences mathématiques. On y retrouve l'une des idées maîtresses des Doutes sur l'astronomie : «L'astronomie a son origine en Chine et a été transmise vers l'Extrême-Occident.» Cependant, alors que Mei cite en exemple les empereurs de l'Antiquité pour arguer que l'astronomie impériale doit être confiée à des spécialistes, Kangxi soutient que sa propre maîtrise des sciences mathématiques est le moyen de tenir son rôle d'intermédiaire entre le cosmos et le monde humain ${ }^{70}$. Chacun des deux justifie donc par un recours à l'histoire et à la cosmologie chinoises son goût pour un domaine d'étude assez peu cultivé par les lettrés de leur temps.

L'idée que les savoirs occidentaux sont d'origine chinoise, qui a prévalu tout au long du XvIII ${ }^{\mathrm{e}}$ siècle, s'avère encore plus utile au souverain qu'au savant. Mei l'a utilisée de deux manières. D'une part, il conteste l'assertion des jésuites selon laquelle ce qu'ils introduisent est radicalement nouveau. D'autre part, il souligne qu'on doit prendre le meilleur de chacun des deux systèmes de savoir pour en faire la synthèse, et que l'origine d'un savoir ne doit pas être un critère de choix. Pour Kangxi, cette thèse change la signification de son soutien aux jésuites et à leurs sciences: si ce que ces derniers enseignent provient de Chine, alors au lieu d'imposer un savoir étranger, l'empereur qui les emploie ne fait que restituer les savoirs de l'âge d'or de l'Antiquité qui ont été perdus. C'est donc pour lui une légitimation culturelle forte. Le sujet a dû être longuement discuté entre Kangxi et Mei lors de leur entrevue. Dans le poème qu'il écrit en souvenir de l'honneur insigne qui lui a été fait, Mei Wending note: "La Discussion des triangles de composition impériale dit que les savoirs européens proviennent en réalité des méthodes chinoises. Que les paroles du souverain sont grandes ${ }^{71}$ !» Et dans les années qui suivent l'audience, il travaille à un Supplément aux doutes sur l'astronomie (Lixue yiwen $b u$ ); il y accumule les arguments en faveur de son idée, que Kangxi lui a fait l'honneur d'adopter ${ }^{72}$.

70. Jami 2012: 249-250.

71. Mei $1995: 327$.

72. Han 1996: 441. 
Cette audience est pour Mei Wending une consécration; au-delà de sa personne et de son œuvre, c'est l'existence d'une expertise mathématique parmi les lettrés chinois que l'empereur reconnaît à travers lui. Il y a en fait légitimation multiple: l'audience avec Kangxi augmente certainement la renommée de Mei ; ce dernier apporte au goût de l'empereur pour les sciences et à sa politique dans ce domaine la reconnaissance d'un expert renommé de la communauté savante chinoise. Et cette promotion mutuelle contribue aussi à rehausser le prestige des sciences mathématiques.

La rencontre marque aussi un tournant dans la politique impériale. Dès 1703, Kangxi exprime son mécontentement devant l'augmentation, à ses yeux désordonnée, du nombre de missionnaires et de chrétiens chinois au Jiangnan. Quelques mois après sa rencontre avec Mei Wending, un légat du pape informe l'empereur de l'interdiction faite par le pape aux chrétiens chinois de participer au culte des ancêtres. À partir de là, Kangxi prend ostensiblement ses distances vis-à-vis des jésuites, qu'il ne tolère désormais «que pour utiliser leurs compétences $^{73}{ }^{\gg}$. Ayant reconnu, à travers la personne de Mei, les compétences de certains lettrés chinois en sciences mathématiques, il cherche de plus en plus activement à réunir à la cour de tels lettrés. En 1712 se tient un examen extraordinaire, qui vise à recruter le personnel d'un Office des mathématiques (Suanxue guan) conçu sur le modèle de l'Office de l'Histoire des Ming, pour travailler à la compilation d'un ouvrage impérial d'astronomie, de mathématiques et d'harmonie musicale. C'est là que s'opère la rencontre entre expertise impériale et expertise lettrée dans ces domaines. En effet, les lauréats de l'examen son placés sous la supervision du troisième fils de Kangxi, ancien élève d'Antoine Thomas, et ils travaillent, sans aucune collaboration avec des jésuites, à partir des manuels produits essentiellement par Thomas pour l'empereur à partir des années 1690. D’un autre côté, les membres les plus en vue de l'Office n'ont pas passé l'examen. Ce sont presque tous les élèves auxquels Mei Wending a enseigné les deux manuels impériaux une dizaine d'années plus tôt à Baoding: outre son petit-fils Mei Juecheng, l'un des deux compilateurs en chef, on retrouve Wang Lansheng, Wei Tingzhen et Wu Yongxi parmi les compilateurs. L'autre compilateur en chef, He Guozong (? -1666), est issu d'une famille d'astronomes professionnels : son père est fonctionnaire au Bureau de l'astronomie. Les trois ouvrages qui sont imprimés tout à la fin du règne de Kangxi sous le titre général Sources de l'harmonie musicale et de l'astronomie, de composition impériale (Yuzhi lüli yuanyuan), et officiellement promulgués par son successeur Yongzheng (r. 1723-1735) en 1724, sont ainsi le fruit d'une appropriation des savoirs des jésuites enseignés à Kangxi par les

73. Jami 2012: 255 . 
deux groupes qui étaient versés dans ces domaines : fonctionnaires astronomes et lettrés spécialistes ${ }^{74}$.

Mei Wending, jugé «malheureusement trop âgé » par Kangxi ${ }^{75}$, ne participe pas au travail de compilation. La reconnaissance suprême qui lui est échue permet en premier lieu l'impression de plusieurs de ses ouvrages en 1706, toujours àl'initiative de Li Guangdi. Cette même année, Mei rentre à Xuancheng, où il continue à recevoir de nombreux visiteurs. La maison familiale, isolée en pleine campagne, est devenue un lieu de savoir mathématique, et semble l'être restée jusqu'à sa mort en 1721. En revanche, la carrière de son petit-fils Mei Juecheng doit beaucoup à cette reconnaissance. Formé par son grandpère, Juecheng sera promu au grade de lauréat de l'examen provincial (juren) puis de lauréat de l'examen métropolitain (jinshi) pour sa contribution aux Sources de l'harmonie musicale et de l'astronomie. Les postes auxquels il est nommé après l'achèvement de l'ouvrage, cependant, ne font pas appel à son expertise en sciences mathématiques. L'achèvement de l'ouvrage impérial, qui signifie la fermeture de l'Office créé pour sa compilation, coïncide avec la mort de Kangxi. L'institution créée pour l'élaboration des savoirs impériaux dans ces domaines techniques n'avait pas vocation à la pérennité. En revanche, les œuvres de Mei Wending, dont une collection est publiée en 1723, une seconde en 1761, seront lues et commentées tout au long du XVIII siècle dans la communauté savante ${ }^{76}$. Ainsi la reconnaissance dont a bénéficié Mei n'a pas ouvert la voie à une professionnalisation du lettré-mathématicien dont il est en quelque sorte le prototype, mais à une intégration des sciences mathématiques parmi les domaines dans lesquels les savants chinois pouvaient être spécialisés. De ce point de vue, le passage par la capitale et par les institutions impériales représente un détour crucial dont la fonction a été de rehausser le prestige d'un individu et d'un domaine d'étude. Le Jiangnan, où Mei Wending est né et où il a passé presque toute sa vie, reste le lieu de cette intégration.

La mobilité géographique de Mei Wending dessine un itinéraire typique de lettré de son époque: de chez lui à la capitale provinciale, suivant le parcours des examens impériaux; à la capitale puis dans une autre capitale provinciale, à la suite d'un haut fonctionnaire. Si l'on s'en tient à la caractérisation des lieux où il a résidé suivant le système de la fonction publique, il est un exemple de ce que pouvait être la carrière de la majorité des lettrés, ceux qui échouaient

74. Jami 2012: 364-378.

75. Jami 2012: 253 .

76. Voir ci-dessus note 2. 
aux examens. Ils suivaient souvent un haut fonctionnaire en tant qu'hôtes ( $k e$ ) ou secrétaires particuliers (тиуои).

Nankin, la ville où il passe l'examen provincial, est pour Mei Wending le premier lieu d'intégration dans la communauté savante en tant que spécialiste d'un domaine assez peu étudié dans cette communauté. L'accès aux livres et surtout la diffusion de ses propres écrits s'appuient moins sur le commerce de l'imprimé que sur la sociabilité entre lettrés, parmi lesquels se dessinent deux groupes: un petit nombre de spécialistes qui partagent son intérêt (ce sont les lecteurs de la Discussion des tableaux rectangulaires), et un groupe plus large, dont le statut social et les moyens financiers sont en général supérieurs aux siens, qui exercent envers lui une forme de patronage; ce second groupe est destinataire du grand nombre d'écrits qui relèvent de la vulgarisation ou de l'enseignement (comme les Réglettes de Wu'an). Dans ce contexte, l'impression de ses livres semble avoir servi autant, sinon plus, à la promotion de leur auteur qu'à la circulation des savoirs qu'il a produits - mais ces deux aspects sont peut-être indissociables.

À ce stade, une question doit être posée: qu'y a-t-il de spécifique aux sciences mathématiques dans l'itinéraire, tant géographique que social, de Mei Wending et la dynamique de circulation des savoirs qui le sous-tend? Il semble que la réponse pourrait être dans l'un des lieux où l'a mené sa quête des savoirs spécialisés: Pékin. Si Pékin était un lieu de savoir privilégié pour les sciences mathématiques, c'est d'une part à cause de l'importance de l'astronomie comme science d'État, raison structurelle, et d'autre part en raison du goût de l'empereur pour ces sciences, raison conjoncturelle. L'orientation de l'activité scientifique des jésuites vers la cour s'explique par la première de ces deux causes, et elle est à l'origine de la seconde. En d'autres termes, la mise des sciences occidentales au service du pouvoir mandchou a accentué la polarisation de l'activité dans ce domaine en direction de la cour. L'itinéraire de Mei Wending peut alors être lu comme une démarche d'appropriation des savoirs occidentaux pour la communauté lettrée chinoise et comme l'intégration de ceux-ci dans le corps des savoirs chinois. 


\section{BIBLIOGRAPHIE}

ArRault Alain (2002). Shao Yong (1012-1077), poète et cosmologue. Paris, Collège de France, Institut des Hautes Études Chinoises.

Ben-Dor Benite Zvi (2005). The Dao of Muhammad: A Cultural History of Muslims in Late Imperial China. Cambridge (Mass.), Harvard University Asia Center.

Cervera José Antonio (2011). Las varillas de Neper en China. Giacomo Rho, S. J. (1592-1638) y su trabajo com matemático en Beijing. Mexico, El Colegio de México.

CHEMLA Karine et Guo Shuchun (2004). Les Neuf Chapitres : le classique mathématique de la Chine ancienne et ses commentaires. Paris, Dunod.

Chu Pingyi (1994). «Technical Knowledge, Cultural Practices and Social Boundaries: Wan-nan Scholars and the Recasting of Jesuit Astronomy. 1600-1800», PhD dissertation. Los Angeles, UCLA.

Elman Benjamin A. (2000). A Cultural History of Civil Examinations in Late Imperial China. Berkeley, University of California Press.

Engelfriet Peter M. (1998) Euclid in China: The Genesis of the First Chinese Translation of Euclid's Elements, books I-VI (Jihe yuanben, Beijing, 1607) and its Reception up to 1723. Leyde/Boston, Brill.

Engelfriet Peter (1998a). «The Transmission of Western Scientific Knowledge in Nanjing during the Shunzhi Reign». Communication au colloque international Europe in China III. Berlin, 1998.

HAN Qi 韓琦 (1996). «Junzhu he buyi zhijian: Li Guangdi zai Kangxi shidai de huodong ji qi dui kexue de yingxiang 君主和布衣之間: 李光地在康熙時代的 活動及其對科學的影響.» Qinghua xuebao 清華學報, vol. 26, nº 4: 421-445.

НаSнiмото Keizō 橋本敬造 (1970). «Bai Buntei no rekisan gaku - Kōki nenkan no tenmon rekisangaku 梅文鼎の暦算学一康熙年間の天文暦算学.» Tōho gakuhō 東方学報, vol. 41: 491-518.

HаSнімото Keizō 橋本敬造 (1973). «Bai Buntei no sūgaku kenkyū 梅文鼎の数学研 究.»Tōhō gakuhō 東方学報, vol. 44: 233-279.

JAMI Catherine (1994). «History of mathematics in Mei Wending's (1633-1721) work.» Historia Scientiarum, vol. 53: 157-172.

JAMI Catherine (2004). «Légitimité dynastique et reconstruction des sciences en Chine au XVII ${ }^{\mathrm{e}}$ siècle: Mei Wending (1633-1721).» Annales, vol. 59, $\mathrm{n}^{\circ}$ 4: 701-727.

JAMI, Catherine (2012). The Emperor's New Mathematics. Western Learning and Imperial Authority during the Kangxi Reign (1662-1722). Oxford, Oxford University Press.

Leсомте Louis (1990). Un jésuite à Pékin: nouveaux mémoires sur l'état présent de la Chine 1687-1692. Édité par Frédérique Touboul-Bayeure. Paris, Phébus.

LI Di 李迪 (2006). Mei Wending pingzhuan 梅文鼎评传. Nanjing, Nanjing daxue chubanshe.

Li Yan 李俨 (1998). Mei Wending nianpu 梅文鼎年谱》. In Li Yan 李俨 et Qian Baocong 钱宝琮, Li Yan Qian Baocong kexueshi quanji 李俨钱宝琮科学史全 集. Shenyang, Liaoning jiaoyu chubanshe, vol. 7: 515-545. 
Magone Rui (2001). «Once every three years: people and papers at the Metropolitan examination of 1685.» Thèse de doctorat, Freie Universität Berlin.

McDermott Joseph (2006). A Social History of the Chinese Book. Hong Kong, Hong Kong University Press.

MARTZlofF Jean-Claude (1981). Recherches sur l'œuvre mathématique de Mei Wending (1633-1721). Paris, Collège de France, Institut des Hautes Études Chinoises.

MSCSJY: Meishi congshu jiyao 梅氏叢書輯要, 1761 .

Pardies Ignace Gaston S. J. (1671). Elémens de géométrie. Paris, Sébastien MabreCramoisy.

SHI Yunli (2007). «Nikolaus Smogulecki and Xue Fengzuo's Tianbu zhenyuan : its production, publication, and reception.» East Asian Science, Technology and Medicine, vol. 27: 63-126.

SKQS: Yingyin Wenyuange Siku quanshu 景印文淵閣四庫全書. 1500 vols. Taipei: Taibei shangwu yinshuguan, 1986.

TIAN Miao 田 丞 et ZHANG Baichun 张柏春 (2006). «Mei Wending 'Yuanxi qiqi tushuo luzui'zhu yanjiu 梅文鼎《远西奇器图说录最》注研究.》Zhongguo kejishi $z a z h i$ 中国科技史杂志, vol. 27, $\mathrm{n}^{\circ} 4: 330-339$.

Tong Qingjun 童慶鈞 et FENG Lisheng 馮立昇 (2007). «Mei Wending 'Zhongxi suanxue tong'tanyuan 梅文鼎《中西算學通》探原.»Nei Menggu shifan daxue xuebao (ziran kexue hanwen ban) 內蒙古師範大學學報(然科學漢文版), vol. $36, n^{\circ} 6: 717-721$.

WAKEMAN Frederic Jr. (1985). The Great Enterprise: The Manchu Reconstruction of Imperial Order in Seventeenth-Century China, 2 vol. Berkeley, University of California Press.

WiLHelm Hellmut (1951). «The Po-hsuëh hung-ju examination of 1679.» Journal of the American Oriental Society, vol. 71 : 60-76.

The Ricci Roundtable, http://ricci.rt.usfca.edu/index.html

\section{GLOSSAIRE}

Baoding 保定

Bei Zhili 北直隸

Bili gui jie 比例規解

buzhu 補註

Chen Wance 陳萬策

Cheng Yi 程頣

chi 尺

Chongzhen lishu 崇禎曆書

chousuan 籌算

Chousuan 籌算

Fang Yizhi 方以智 
Fang Zhongtong 方中通

Fangcheng lun 方程論

Fangtian tongfa 方田通法

fenye 分野

$\mathrm{Gu}$ Yanwu 顧炎武

He Guozong 何國宗

Hebei 河北

Henan 河南

Huang Yuji 皇虞稷

Huang Zongxi 黃宗義

huihui 回回

Huihui ke 回回科

Jiangnan tongzhi 江南通志

Jiangnan 江南

Jiangning 江寧

Jiayu 嘉魚

Jihe yuanben 幾何原本

jinshi 進士

Jiuzhang suanshu 九章算術

juan 卷

juren 舉人

$k e$ 客

$\mathrm{Li} \mathrm{Di}$ 李迪

Li Dingzheng 李鼎徵

Li Guangdi 李光地

Li Zhonglun 李鍾倫

Liu Dun 劉鈍

Lixue huitong 曆學會同

Lixue pianzhi 曆學駢枝

Lixue yiwen bu 暦學疑問補

Lixue yiwen 曆學疑問

Lizhi zhuiyan 曆志贅言

Ma Decheng 馬德稱

Mao Qike 毛奇可

Mei Juecheng 梅款成

Mei Wending 梅文鼎

Mei Wending jinian guan 梅文鼎紀念館

Meishi Congshu jiyao 梅氏叢書輯要

Mingshi guan 明史館

Mingshi lizhi nigao 明史曆志擬稿 
Mingshi 明史

muyou 幕友

Nan Zhili 南直隸

Nanjing 南京

nanxun 南巡

Ni Guanhu 倪觀湖

$n i a n p u$ 年譜

Ningguo 寧國

Ningguo fuzhi 寧國府志

Pan Lei 潘末

Ping li ding sancha xiangshuo 平立定三差詳說

pudijin 舖地錦

Pushuting ji 曝書亭集

$q i$ 氣

Qintianjian 欽天監

Quanzhou 泉州

$r u$ 儒

Sanjiaofa juyao 三角法舉要

Sanjiaoxing tuisuanfa lun 三角形推算法論

Shaanxi 陝西

Shi Runzhang 施閏章

Shudu yan 數度衍

Siku quanshu 四庫全書

Suanfa tongzong 算法統宗

Suanfa yuanben 算法原本

Suanxue guan 算學館

Tianbu zhenyuan 天步真原

Tongwen suanzhi 同文算指

Wang Lansheng 王蘭生

Wei Tingzhen 魏廷珍

wenfang sibao 文房四寶

Wenfeng Meishi zongpu 文峰梅氏宗譜

wenji 文集

Wenmi 文鼏

Wennai 文倪

$\mathrm{Wu}$ Yongxi 吳用錫

Wu'an chousuan 勿庵籌算

Wu'an lisuan quanshu 勿庵曆算全書

Wu'an lisuan shuji 勿庵曆算書記

Wu'an lisuan shumu 勿庵曆算書目 
$x i$ 西

xiangshu 象數

xiucai 秀才

xixue 西學

Xiyang xinfa lishu 西洋新法曆書

Xu Guangqi 徐光啓

Xuancheng xianzhi 宣城縣誌

Xuancheng 宣城

Xue Fengzuo 薛鳳祚

yao liang pingmian $f a$ 遙量平面法

Yijing 易經

Yiyan 以燕

$\mathrm{Yu}$ 俞

Yuzhi lüli yuanyuan 御製律曆淵源

Zhang Wenzhen ji 張文貞集

zhang yuan xueshi 掌院學士

Zhang Yushu 張玉書

Zhili 直隸

zhong 中

Zhongxi suanxue tong 中西算學通

Zhu Xi 朱喜

Zhu Yizun 朱彝尊

$z i$ 字 